\title{
PROFESORADO Y FAMILIAS. ACTORES SIN GUION
}

Joaquín Giró Miranda

Sergio Andrés Cabello

Universidad de La Rioja

\begin{abstract}
RESUMEN: El sistema educativo se encuentra en una constante transformación por los cambios que se dan en la sociedad. Sin embargo, el sistema educativo no va a la misma velocidad que la sociedad y tiene que adaptarse a los mismos. Uno de los más importantes es la irrupción de las Tecnologías de la Información y la Comunicación (TIC), que incide en el conjunto de la educación. Este escenario afecta a toda la comunidad educativa, que se encuentra en una situación de incertidumbre. Las escuelas y los profesores asumen nuevas funciones y las familias aumentan sus demandas sobre el sistema educativo. Este artículo analiza la situación de docentes y madres y padres ante estos cambios, que también incide en la relación entre ellos a través de nuevos canales de comunicación, y se basa en un estudio cualitativo en centros educativos que cuentan con experiencias de éxito en la participación de las familias en la escuela.
\end{abstract}

PALABRAS CLAVE: Profesorado, familias, sistema educativo, funciones de la educación, participación de las familias en la escuela.

\section{TEACHING STAFF AND FAMILIES. ACTORS WITHOUT SCREENPLAY}

\footnotetext{
ABSTRACT: The educational system is in a constant transformation because of changes happening in society. Nevertheless, educational system does not go at the same speed than society and it has to adapt to them. One of the most important changes is the irruption of the Information and Communication Technology (ICT) influencing education as a whole. This scenario affects the entire educational community that is in a situation of uncertainty. Schools and teachers assume new functions and families increase their demands on the educational system. This article analyzes the situation of teachers, and parents in face of these changes, which also affects the relation between them through
} 
new channels of communication, and is based in a qualitative research in schools with successful experiences in the involvement of families in schools.

KEYWORDS: Faculty staff, families, educational system, functions of education, family involvement in school.

Recibido: 21/10/2017

Aceptado: 21/11/2017

Correspondencia: Sergio Andrés Cabello, Universidad de La Rioja, Departamento de Ciencias Humanas, C/ Luis de Ulloa, Edificio Vives, 26004 Logroño, La Rioja. Email: sergio.andres@unirioja.es.

\section{INTRODUCCION}

Los cambios económicos, sociológicos, culturales y tecnológicos que se han producido en estas últimas décadas, Ilevan incorporados también cambios en el quehacer educativo, el cual, no lo olvidemos, se ha universalizado desde los años ochenta del siglo pasado. Estos cambios no hacen sino incidir en los determinantes pasos dados en los procesos de socialización y educación. No hay más que reconocer la existencia de un modelo económico basado en la globalización de los mercados financieros y la aceptación de un sistema laboral flexible y cambiante al albur de las necesidades productivas, para entender la necesidad de una educación igualmente cambiante, no basada en la transmisión de saberes, sino en el aprendizaje de tareas tendentes a la creación de conocimiento. Los contenidos escolares se van a interrelacionar más con el mundo laboral, primando la competencia sobre el conocimiento y la información y la creatividad sobre la reproducción.

Sin embargo, el sistema educativo parece ir a la zaga de los cambios sociales, y ahora mismo está posicionado en función del sistema económico, del trabajo y el empleo, intentando adaptarse sin cubrirlas a las demandas del mercado laboral, por una inercia de décadas que le impiden adaptarse a los cambios estructurales que demanda una sociedad tan volátil. Además, el mercado laboral tampoco es eficiente y poco puede orientar al sistema educativo sobre sus intereses.

Mientras el sistema productivo ha evolucionado rápidamente, la escuela ha seguido manteniendo un currículo nada adecuado a los cambios sociales que se han producido en los últimos años, que exigen una formación permanente para la vida y no sólo para el trabajo. Es como si el sistema educativo olvidara que además de procurar mano de obra cualificada y la empleabilidad del alumnado, también debería pensar en la construcción del ciudadano y en la formación integral de las personas en todas sus dimensiones, de modo que les permitiera gozar de las relaciones con los demás.

Pese a que los cambios sociales han dado lugar a que la escuela esté asumiendo nuevas funciones que en el pasado se encontraban depositadas en la familia y en la iglesia, la sociedad acaba responsabilizando de todo a la escuela, empujándola hacia una crisis por las condiciones cambiantes de sus funciones. 
Mientras en el pasado la sociedad tenía unos valores muy homogéneos que eran más fáciles de transmitir por las familias o la escuela a las jóvenes generaciones socializándolas, los roles se transmiten en la actualidad no sólo a través de padres y maestros, sino también por los medios de comunicación y las nuevas tecnologías que manejan una información inabarcable para los primeros. En consecuencia, los cambios de paradigma cultural hacia lo audiovisual y lo instantáneo son parte de ese dominio ejercido por las nuevas tecnologías de la información, las cuales se acompañan por una cierta desidia cooperativa de las familias, así como la inevitable devaluación de la figura de maestros y profesores, desdibujando los roles precisos que les acompañaron durante la segunda mitad del siglo XX y que en la actualidad se mueve en un bucle de crisis permanente.

Así, desde parte de la institución y del profesorado "existe un elevado consenso en considerar que se da una excesiva delegación de responsabilidades hacia la escuela y el profesorado en todo lo pertinente a la educación de los hijos, y que las familias deberían asumir su rol educativo y la escuela, por su parte, las responsabilidades formativo-instructivas" (Giró et al., 2014, p. 84), por lo que demandan de las familias "mayor implicación en el proceso educativo, tarea que consideran que ha sido derivada al profesorado, sobrecargándolo con múltiples tareas a realizar" (Doncel, 2012, p. 498).

Si partimos de la heterogeneidad y diversidad existente entre el profesorado, que acumula imágenes y representaciones construidas sobre una gran diversidad de familias, entenderemos de la dificultad de establecer modelos o tipologías de profesorado pues muchas de sus representaciones sociales en no pocas ocasiones derivan de experiencias previas, que pueden no ser positivas, y que van a estructurar la relación con alumnos, familias y entorno educativo.

Además, también se producen límites a las familias, ya que el profesorado define un espacio (el aula) y un ámbito (el pedagógico) como propio y casi "exclusivo", dando lugar a que "lo más frecuente es que existan resistencias hacia la participación por parte del profesorado, que, con el argumento del profesionalismo, percibe la colaboración de los padres como una injerencia en el trabajo docente" (Consejo Escolar del Estado, 2014, p. 15).

En definitiva, tópicos y lugares comunes que funcionarían como "una profecía que se cumple a sí misma", y que derivarían hacia la baja implicación de las familias, a su acomodación a un rol secundario, a la individualización de las relaciones entre el profesorado y las familias, y hacia unos objetivos basados en los resultados académicos, especialmente en Secundaria (Giró et al., 2014).

\section{Metodología}

Los resultados presentados en este artículo sobre las relaciones profesorado y familias proceden del Proyecto de Investigación "Familias y escuelas. Discursos y prácticas cotidianas sobre la participación en la educación obligatoria" (referencia: EDU2012-32657) de la convocatoria 2012 del Subprograma de Proyectos de Investigación Fundamental no Orientada del Ministerio de Economía y Competitividad. Este proyecto ha analizado las relaciones entre familias y escuelas con- 
siderando, entre otros aspectos, el discurso de familias e integrantes de centros escolares acerca de la participación en cuatro Comunidades Autónomas: Cataluña, Aragón, Islas Baleares y La Rioja.

Un equipo interdisciplinar, formado por sociólogos de la educación, pedagogos, trabajadores sociales y psicólogos, llevó a cabo un extenso trabajo de campo cualitativo en treinta y dos centros de estas regiones, realizando una etnografía de cada uno de ellos basada fundamentalmente en entrevistas en profundidad con equipos directivos, docentes, madres y padres, personal no docente de los centros y otros informantes.

Más concretamente, el proyecto de investigación del que surgen los principales resultados se inició con la búsqueda y análisis de la legislación específica en cada comunidad autónoma y la investigación realizada sobre participación de las familias en la escuela. Además, se realizó una primera fase de trabajo empírico con entrevistas en profundidad a 46 personas (representantes políticos; representantes de las administraciones autonómicas/locales educativas; representantes del Consejo Escolar autonómico; representantes de federaciones de Asociaciones de Madres y Padres de Alumnos; representantes de sindicatos; representantes de movimientos sociales/ educativos y representantes de movimientos de renovación pedagógica), que podían ayudar a definir claramente la evolución que ha realizado la participación de los agentes educativos en las diferentes comunidades autónomas, así como presentar un retrato de la situación actual.

Por otro lado, en una segunda fase la investigación consistió en una etnografía en 32 centros escolares e institutos con una duración mínima de 8 meses en cada uno de ellos, diferenciando por perfil de titularidad de centro y nivel educativo que imparte. Además, en cada comunidad autónoma se ha realizado etnografía en al menos una escuela rural y una comunidad de aprendizaje. Dentro de la muestra de centros seleccionados se contó con todas las tipologías posibles: Centros de Educación Infantil y Primaria (CEIPs), Institutos de Educación Secundaria (IES), centros concertados, centros rurales y escuelas unitarias, y centros que experimentan en comunidades de aprendizaje.

De este modo se ha conseguido un gran cúmulo de información directa sobre la que se basa la redacción de este artículo.

\section{La implicación de familias y docentes}

La comunidad educativa está formada por el conjunto de los colectivos y personas que participan de la escuela y su entorno. Estudiantes, profesores, equipos directivos, personal de la administración vinculado directa o indirectamente al ámbito educativo, y padres y madres conforman una comunidad con el fin último de alcanzar el éxito escolar. Todos ellos son corresponsables del mismo y juegan un papel determinante, aunque diferentes estudios indican que la implicación de los padres y madres en la educación es más relevante (Fernández-Enguita, 2007; San Fabián, 2001; Garreta, 2003, 2008). Sin embargo, está lejos de ser todo lo exitosa que se desearía, ya que las familias todavía cuentan con una participación reducida, especialmente en lo que hace referencia a los ámbitos más institucionales y formales, como por ejemplo Asociaciones de Madres y Padres (AMPAs) y Consejo Escolar (Fernández Enguita, 2006 y 2007; Garreta, 2003, 2008, 2017; Feito, 2007; Garreta 
y Llevot, 2007; Bernard y Llevot, 2016). No obstante, hay una serie de aspectos que son determinantes y que demuestran que favorecen la participación de padres y madres en la escuela, cuyas motivaciones, predisposición y carácter dependen en gran medida de las interrelaciones que generan y construyen. Además, equipos directivos, profesores, padres y madres son transitorios, y en algunos casos su implicación con la institución escolar dura un periodo muy limitado.

La implicación de los padres en las escuelas es uno de los principales aspectos de la llamada comunidad educativa y de los procesos de apertura de las instituciones educativas a la sociedad. A lo largo de las últimas décadas se ha incrementado esta implicación a través de diferentes canales, reconocidos institucional y legislativamente. Sin embargo, ofrece numerosas dificultades y no ha conseguido ser del todo exitosa, pues nos encontramos ante una inabarcable variedad de posturas y actitudes ante la participación de las familias en las escuelas, aunque observamos elementos comunes (Rivas et al., 2011; Usategui y Del Valle, 2009; San Fabián, 2001). Sin embargo, en todos los estudios sobre la implicación de madres y padres, existe un déficit en ese papel, fundamental, que desempeña el profesorado (Gomila y Pascual, 2015), y que investigaciones recientes comienzan a paliar, partiendo de la base de que la cooperación entre los padres y la escuela es también un fenómeno reciente (Llevot y Bernard, 2015).

En los últimos años, con las transformaciones de la sociedad en sus diversos ámbitos, se ha producido una complejización de los escenarios de participación, a la vez que surgen nuevos canales de comunicación (especialmente a través de las Tecnologías de la Información y Comunicación) (Garreta y Llevot, 2015). Y es que escuela y sociedad no son dos realidades indisociables, al contrario, no se explican la una sin la otra.

Si tanto el profesorado como las familias son dos actores colectivos en el interior de la comunidad educativa, y sin cuyo concurso no puede hablarse de participación en la escuela, la necesidad de un trabajo conjunto y coordinado entre los distintos agentes sociales, tal como lo ponen de manifiesto Garreta (2009, 2008, 2007), Pereda (2006), Bolívar (2006), Epstein (2001) entre otros, resulta fundamental pensando en el desarrollo educativo de los niños y adolescentes.

Así pues, los dos colectivos están condenados a entenderse mientras la principal motivación e interés de ambos sea que hijos/alumnos consigan el mayor rendimiento escolar, si bien la relación entre docentes y familias está marcada por una elevada desconfianza del profesorado hacia ellas que, en no pocas ocasiones, lleva a la generación de barreras y límites. Muchos profesores apuntan en su discurso que las barreras se levantan en los aspectos académicos y en el funcionamiento del aula, espacio que estaría consagrado en exclusividad a la función docente.

\section{Funciones del profesorado}

Atendiendo a los nuevos roles que se adscriben a los actores educativos, concretamente al profesorado, escuchamos lo que parece ser un discurso hegemónico acerca de las dos funciones a que obliga su práctica docente, como son la de enseñar académicamente lo que indica el currículo a través de las diferentes materias, pero también formar en valores, en modos de relacionarse socialmente y, en última instancia, formar ciudadanos. Entre estas dos funciones establecidas como primarias por el 
profesorado, ser transmisor de conocimientos y educador o socializador para el desarrollo integral de sus alumnos, hay profesores que señalan los problemas para aunar ambas por la dificultad de evaluar la madurez emocional o el pensamiento crítico de sus alumnos, inclinándose por la búsqueda de equilibrio entre las dos funciones.

En general, hay una percepción sostenida por el profesorado, y es que la escuela tan sólo cumple la función de guarda y custodia de niños y adolescentes junto a la transmisión de conocimientos, pese a que ellos representan los roles de controladores, mediadores, preparadores, motivadores y diseñadores de estrategias pedagógicas según su propia construcción discursiva. Toda una panoplia de responsabilidades insertas en sus funciones y construidas a través del campo de representación que es su discurso.

Esta percepción del profesorado es cierto que responde a lugares comunes que señalan a la escuela en su función de guarda y custodia, pero, en la práctica, la responsabilidad del profesorado que viene sustentada en la autoridad que determina la jerarquía escolar, se asume a partir del control ejercido en el aula y del sometimiento del alumnado a un orden que signifique la construcción de un clima sobre el que se pueda llevar a cabo el proceso de enseñanza aprendizaje. Hay profesores que lamentan la pérdida de autoridad, del respeto que se les tenía en el pasado, y de que madres y padres cuestionen su labor. Sin embargo, la mayoría de los docentes asume que este proceso es imparable, que las relaciones ya no se pueden basar en ese rol de autoridad tan definido en lo autoritario, y que el nivel formativo y cultural de madres y padres ha crecido en las dos últimas décadas, demandando relaciones de igual a igual y sin complejos, a diferencia de las generaciones de sus padres (Usategui y Del Valle, 2009).

Los límites, el profesor es el que manda, entre comillas, porque no es el que manda pero sí es el que puede decir hasta aquí se puede llegar y, el que pase de ahí, pues...no, porque si no, pasa lo que está pasando últimamente, que los padres siempre dicen que sus hijos tienen razón y se le está quitando la autoridad (CPUPRPLR14AI)

Ahora hay padres que tienen un nivel educativo superior a los profesores. He escuchado comentarios de 'cómo habla, cómo escribe'. Claro, es que igual estábamos acostumbrados a unos padres que tenían un nivel educativo... pero es que ahora hay padres universitarios, abogados, economistas (IPUPS2LR14AII)

En este sentido, penetran y avanzan en sus funciones más allá de la mera transmisión del currículo, pues en el límite difuso entre funciones de padres y de profesores, se encuentra el asumir y aplicar normas básicas de comportamiento y convivencia de acuerdo a la autoridad con que se revisten $y$, donde algunos demuestran capacidad de mediación en los conflictos, aunque muestren reticencias hacia la asunción de este tipo de responsabilidades.

Las familias tienen una visión de la enseñanza muy sesgada, que ven la enseñanza, se fijan en cosas muy puntuales. Y, sin embargo, no son conscientes de otros aspectos del proceso de enseñanza-aprendizaje (CPUPP1LR14AII)

Este discurso sobre las funciones de guarda y custodia se ha elevado de forma hegemónica tanto entre los más adultos como entre el profesorado joven, desde que adoptaron en sus relaciones pedagógicas realizar no sólo la transmisión del currículo 
sino la motivación y orientación para con el devenir socio profesional de los alumnos, aspectos que implican una función de socialización que, no obstante, no puede ser ajena al entorno familiar y social en el que se mueven los jóvenes.

Para conseguirlo necesitan construir la motivación de un alumnado supuestamente desmotivado por la irrupción de Internet en el proceso de enseñanza aprendizaje, donde los estudiantes creen haber encontrado todo lo necesario sin el recurso del profesorado, el cual según su entender tendría una mayor dedicación a medir los resultados del aprendizaje a través de pruebas como los exámenes u otros sistemas de evaluación, más que a enseñar materias curriculares a las que el alumnado no encuentra utilidad funcional.

Sin embargo, y como ya hemos entrevisto, el aula o el centro no viven sus experiencias de modo extraño a cuanto ocurre en la sociedad, de modo que, si ésta tiende hacia la desigualdad, el sistema educativo se manifestará de la misma forma especializándose en la excelencia del alumnado con más posibilidades (aspecto que se valora a través del empuje que se ofrece a los centros con altas capacidades en detrimento de aquellos centros que acogen a los sectores más desfavorecidos). De ahí que no sea novedoso el interés familiar por aquellos centros concertados o privados significados por los resultados académicos o por las trayectorias profesionales de éxito de sus exalumnos. Así pues, las diferencias entre entornos y centros educativos pueden ser tan decisivas como las diferencias propias de la diversidad familiar.

La diversidad de contextos y entornos educativos, las diferencias entre las distintas etapas educativas o según titularidad de centros, así como la propia heterogeneidad del profesorado no permite que éstos se reconozcan como un colectivo profesional pese a disponer de un discurso corporativo que señala elementos que le han sido detraídos o que se han incorporado sin su consentimiento en la práctica docente, encontrando en la administración, los equipos directivos y la diversidad de familias, los actores educativos que se lo impiden.

Efectivamente, el profesorado es un agente educativo que no dispone de un estatuto profesional que delimite sus funciones y sus responsabilidades y, por ello, se encuentra sometido al albur de los cambios legislativos en materia educativa que le imponen formas de proceder que modifican su práctica docente. Son los cambios en la legislación, pero también son las consecuencias del largo periodo de crisis que mediatiza sus intervenciones, al estar configuradas por ciertas representaciones de lo que debe ser un buen docente, sin que la práctica de la transmisión del currículo se adecue coherentemente con ese discurso.

Se parte de un sistema educativo que legislativamente promueve la igualdad, al tiempo que recorta los instrumentos para alcanzarla, tal y como señala el profesorado cuando constata que cada vez debe realizar más funciones que las estrictamente docentes, principalmente las burocráticas a través de programaciones, estándares, etc., que no inciden en el proclamado cambio educativo. Tampoco el sistema (en el caso de la LOMCE) ha sabido adaptar los centros donde se llevan a cabo los procesos de enseñanza aprendizaje. De hecho, los centros no pueden ofertar toda la rama de optativas y de asignaturas que, en principio se pierden y, sin embargo, se podrían dar.

Finalmente, otra de las cuestiones inherentes al discurso del profesorado es la necesidad de profundizar en su formación para abordar estos cambios que se están 
produciendo en sus funciones, incluida la cuestión de las relaciones con las familias, así como para afrontar las nuevas demandas de la sociedad hacia la escuela en un entorno cada vez más complejo y cambiante (Bernard y Llevot, 2016; Molet et al., 2016; Zapico-Barbeitio et al., 2017; Vecina et al., 2017).

\section{Formar y socializar}

En conjunto todos los problemas antedichos no han impedido el progreso educativo de la sociedad española, pues si bien partíamos de niveles formativos muy bajos, propios de una época donde la educación no era un derecho universal, es cierto que los alumnos de este siglo cuentan con un capital cultural familiar amplio y diverso y, de algún modo, se han arbitrado criterios con los que evaluar y clasificar al alumnado en función de sus capacidades. Además, con los estándares, ya no es un criterio aprobar o suspender, pues habría que concretar lo que demandan las familias, los alumnos y la sociedad, que no necesariamente pasa por exámenes y calificaciones. Los preámbulos de todas las leyes (de la LOE o de la LOMCE), indican cuál es el rol que deben cumplir como educadores, consistente en trabajar y evaluar por competencias (trabajarlas para evaluarlas), porque ser competente es saber hacer.

Hay también profesores que entienden que su función principal es motivar. Esta idea que maneja cierto profesorado proviene de la percepción acerca de su trabajo docente en la que ellos están para extraer, sacar del adolescente todo su potencial para aprehender; son de algún modo los motivadores de los adolescentes que están perdidos o dormidos y no saben escuchar porque desconocen sus capacidades.

No todos piensan así. De hecho, la docencia ya no es extractora de talento y los docentes, mayoritariamente mujeres, se reconocen más como trabajadores que como educadores. Más general en el discurso del profesorado se encuentra la función de educar en el sentido amplio de socializar. La aspiración del profesorado incluye como una de sus responsabilidades, no siempre planificadas o diseñadas ad hoc, la socialización del alumnado como ciudadano, sin menoscabar la educación en los contenidos curriculares (éxito académico), logrando así la humanización y felicidad de los alumnos y evitando su marginalidad o exclusión (fracaso escolar).

Sin embargo, la educación de un país se rige por leyes que necesariamente se redactan con disposiciones y reglamentos, origen de todas las burocracias. La Administración es la responsable de la organización de los distritos escolares donde se lleva a cabo el intercambio y adopción de alumnos, y es la responsable de los recortes en personal y medios con los que asumir las diversas funciones que vinculan al profesorado con las familias.

Las quejas del profesorado se dirigen hacia el carácter cíclico que han tomado estas leyes en función de los cambios de gobierno, porque no se puede olvidar el carácter coercitivo que dispone el poder político con el control de la educación; y se dirigen hacia el modo de publicar leyes educativas que deberían contar con un papel más activo del profesorado, cuando a su vez se les demanda asumir responsabilidades en la búsqueda de un consenso de toda la comunidad educativa. Al respecto nos señalan que hay problemas intrínsecos a la carrera docente, como la movilidad que impide la asunción de responsabilidades y proyecciones de futuro en los centros, por carencia y/o falta de asentamiento del arraigo y el apego a los mismos. 
Otro lugar común en la queja del profesorado sigue siendo la cuestión del prestigio de la profesión, que se sustancia en la poco contrastada comparación con la situación del profesorado de otros países de la UE y en la supuesta baja valoración que hacen los padres y la sociedad española de la práctica docente, pese a que los datos ofrecen machaconamente una perspectiva contraria a ese pensamiento de subestimación (Fernández-Enguita, 2016; Pérez-Díaz y Rodríguez, 2012), y pese a que muchos profesores sí entienden que disponen de una valoración positiva de su trabajo mientras ocurre todo lo contrario con el sistema educativo que adolece de la misma.

Más certeros se muestran aquellos profesores que entienden que el origen de los desajustes entre los objetivos asumidos (capacitación y cualificación profesional de los alumnos) y los resultados conseguidos, se encuentra en la desigualdad existente tanto en el contexto familiar como específicamente a nivel de mercado laboral. Perciben las desigualdades en función de la implicación de las familias, pues un alumno se verá en mejores condiciones para lograr el éxito académico y la conformación de su identidad cuando la familia se involucra en la supervisión y control de las tareas docentes.

Por otra parte, señalan que hay mucha capacitación, pero poca inserción laboral, de modo que la cualificación profesional sólo constituye la acumulación de un título más. Despertar el espíritu crítico con el que desarrollar la capacidad de emprender y tomar decisiones que permitan abordar cualquier profesión con seguridad y confianza, tan sólo constituye un objetivo superior si atendemos a las dificultades de empleo en el mercado laboral.

Efectivamente, cada uno de estos objetivos (preparar, desarrollar al máximo las potencialidades, ayudar a discernir y a distinguir, dotar de habilidades, estrategias y sentido crítico a sus alumnos), de modo que lleguen hasta donde puedan con su diversidad siendo flexibles y adaptándose a las diferentes circunstancias, se asume como una responsabilidad objetiva del profesorado.

También guiarlos y orientarlos en la búsqueda y selección de información; y para llevar a cabo este cúmulo de responsabilidades docentes, el profesorado manifiesta que su rol consiste en dirigir, coordinar y orientar a los alumnos para que desarrollen sus propias potencialidades, maduren y adquieran las competencias y las responsabilidades que no les demandan o que no les ofrecen en sus casas o en sus familias, de modo que no sólo se circunscribe su responsabilidad a la implementación del currículo escolar sino que va un paso más allá asumiendo responsabilidades que se suponen propias de las familias.

Así pues, el otro agente educativo junto al institucional (inspección, ayuntamiento, consejería, legislación, etc.), que el profesorado señala como responsable en la ampliación de sus funciones, está constituido por las familias. Escuela y familias han tenido una tradición construida sobre oposiciones, hasta el punto de que el crecimiento y expansión de la escuela se realiza negando la influencia y la participación de las familias que, en este sentido, siempre han jugado un papel subordinado en una relación propia de profesionales frente a legos. Además, también existen importantes diferencias en el interior de ambos colectivos dada la heterogeneidad de sus componentes, lo cual determinó el resultado y la situación actual de 
las protestas educativas, pues "las contradicciones internas también se producen en un movimiento muy heterogéneo, con actores que cuentan con intereses diversos y con colectivos que tampoco son homogéneos internamente. Es decir, no todo el mundo, ni profesorado ni familias, tiene la misma percepción o sensibilidad" (Giró y Andrés, 2014 p. 640). Y es que cuando hablamos de movilización del profesorado no nos referimos a todo el colectivo, ni siquiera consideramos que sean el mayor número o en la mayoría de los centros, pero si han destacado en las protestas y en las movilizaciones es porque los docentes, principalmente los damnificados por los ajustes, han sabido con sus acciones aunar a toda la comunidad educativa, desde las familias hasta los estudiantes. Claro que, en la actualidad, pese a que madres y padres son peones en manos del profesorado, siguen apareciendo en el discurso hegemónico del profesorado como un colectivo pasivo que hace dejación de sus obligaciones para con los hijos.

Y si bien no todas las familias tienen los mismos recursos, ni la misma capacidad, ni el mismo interés por la educación de sus hijos, es cierto que aquellas familias que supervisan y controlan el rendimiento académico de sus hijos, entendido éste no sólo como la ayuda en los deberes sino como un acompañamiento del trabajo del profesorado, logran mejores resultados académicos para sus hijos que aquellas familias que se desentienden o que no logran acompañar en todas las facetas su desarrollo educativo. Incluso entre las familias está calando el discurso de que aquellas que son más participativas, son las que menos necesitan apoyar a sus hijos en el logro del éxito escolar (Andrés y Giró, 2016). Pero como no sólo se trata de resultados académicos sino también de formación de ciudadanos, el profesorado achaca a las familias la dejación de la que consideran función principal de las mismas: la educación en valores

Las familias, como agentes educativos, deberían completar la actividad docente del profesorado; sin embargo, si no participan en los términos que se les demanda, es en parte por el desencanto hacia la perspectiva que durante décadas iluminó el imaginario social, una perspectiva asentada en la teoría del capital humano, que al no cumplirse en los términos en que se construyó, al no existir una correlación entre estudios y ocupación laboral, entre estudios y empleo, entre estudios y remuneración... produce desilusión, alcanzando ese desencanto al sistema educativo, a la escuela y a los centros como actores protagonistas.

Efectivamente es una cosa de todos, de la familia, de la escuela y de la sociedad, pues cuando se trabaja en un entorno favorable a la educación, éste deja de ser un problema para convertirse en una oportunidad y a este propósito colabora el desarrollo de las TIC y la escuela-red. No obstante, el profesorado reconoce que la heterogeneidad de las familias y sus condicionantes socioeconómicos en un periodo sostenido de crisis obliga a éstos a asumir responsabilidades que en otras condiciones se podrían exigir a las mismas

Ciertamente la situación socioeconómica de muchas familias ha cambiado hasta el punto de situar a uno de cada tres niños en riesgo de pobreza. La conciliación en un mercado laboral desregulado, el desempleo y la búsqueda de recursos con los que aliviar las carencias domésticas de todo tipo y evitar la exclusión, ha llevado a cierta desatención educativa de las familias. A lo largo de estos años, la Educación ha per- 
dido buena parte de aquellas actividades que le convertían en un mecanismo garante de la igualdad de oportunidades. A medidas como la desaparición de los programas de gratuidad de libros de texto, de ayudas y becas, como por ejemplo las destinadas al comedor, se une la práctica eliminación de la educación compensatoria, del profesorado de apoyo, la no renovación de docentes, la pérdida de derechos laborales de los profesores y su precarización, etc.

Pero quizás la asunción de nuevas responsabilidades está más impresa en la mente del profesorado cuando se trata de socialización en normas de comportamiento, creencias, valores y actitudes del alumnado, tradicionalmente entendidas como propias de las familias y subsidiariamente de la iglesia, pese a que la escuela es hija de la iglesia y, por tanto, continuadora de su labor socializadora.

No entienden por qué deben asumir responsabilidades en materia de educación en valores, si es la familia quien debería jugar ese rol sin delegar en la escuela. $\mathrm{Y}$ se muestran contrarios a asumir ciertas responsabilidades cuanto más diversa y difícil es la población escolar. Sin embargo, hay profesores que entienden que su responsabilidad incluye una relación más empática con las familias de modo que les permita entender y conseguir el desarrollo y la madurez de sus alumnos en su heterogeneidad.

Quienes tienen más clara esta función socializadora son aquellos profesores que hablan de crear ciudadanos para el futuro, dotándoles de valores, y prestando atención a la diversidad del alumnado tanto como a la diversidad de situaciones familiares (lengua, cultura, formación, profesión, etc.), pese a la carga de responsabilidad que conlleva.

En la escuela de masas donde se ha perseguido la democratización escolar, nos encontramos con una cultura competitiva que divide, selecciona y otorga beneficios (créditos, títulos), a los más preparados según el lenguaje escolar, que es el de las familias mejor situadas en la estructura social. La relación de opuestos que se establece entre familias-escuelas tiene su base entre las primeras cuando reclaman una atención singular para sus hijos a la vez que demandan un estilo educativo determinado (escuela privada o pública, religiosa o laica, elitista o popular, etc.). Reclaman igualdad escolar y a su vez apoyan estrategias de desigualdad.

Por su parte la dirección o la orientación que nos indica el profesorado en su discurso, tiene mucho que ver con dirigir, marcar $y$, en definitiva, transmitir las materias curriculares que tienen que estudiar, pese a que con el desarrollo de las TIC son los propios alumnos los que se erigen en protagonistas de su aprendizaje, no sólo porque el entorno digital de los hogares les ha permitido socializarse en el mismo antes que la propia escuela desarrolle su propia de utilización de las TIC, sino porque el profesorado es ahora quien tiene que potenciar su formación en nuevas relaciones, comunicaciones, proyectos e innovaciones educativas en dicho entorno. No obstante, la brecha digital entre nativos (alumnos) e inmigrantes (profesores), sigue presente un gran número de variables que determinan la desigualdad educativa y de oportunidades de participación plena en la vida escolar.

Algunos profesores encuentran en un ejercicio de transversalidad de las materias curriculares el medio para conseguir el desarrollo integral del alumnado, incluido el ámbito de la convivencia, y donde los docentes juegan un rol de guías. 
En consecuencia, los profesores son creativos en materia de diseño de estrategias de enseñanza aprendizaje, incluso han perfeccionado y diversificado los métodos educativos integrando las TIC en su aplicación educativa y, además, se han adaptado a las circunstancias exigidas en los cambios legislativos como son los contenidos curriculares o los resultados académicos. Sólo les ha faltado la colaboración de unos alumnos que absortos en las redes no han aprendido a comunicarse, a escuchar, a madurar.

Por otra parte, las múltiples aplicaciones de las nuevas tecnologías que empiezan a introducirse o que ya se han introducido en las aulas, el cambio en el entorno educativo y de las etapas y edades del aprendizaje (que se ha convertido definitivamente en continuo), así como las variadas posibilidades de movilidad real y virtual del estudiante, traen consigo cambios en los conceptos de escuela, educación, trabajo y ocio.

Estos cambios se van a producir, o se están produciendo de un modo azaroso, debido a la existencia de un vacío metodológico ante las nuevas tecnologías. El profesorado desconoce o apenas vislumbra caminos y guías que le permitan afrontar las nuevas tecnologías de una manera adecuada a los objetivos educativos, que se formulan desde las familias y la sociedad para adaptarse a los cambios. Le falta esa formación continua necesaria en el mundo de las TIC.

Pero no son sólo los docentes quienes tienen que adaptarse a los cambios tecnológicos. También las familias deben formarse, reciclarse o adaptarse a los cambios tecnológicos, para que sepan ejercer un control sobre sus hijos en lo que respecta al modo y uso razonable de las TIC.

El profesor no puede quedar atrás en el conocimiento de las nuevas tecnologías aplicadas a la educación y debe formarse permanentemente, pero también los centros deberían dotarse de los medios necesarios para conseguir los objetivos propuestos, porque la realidad que percibe el profesorado es que hay una falta total de coherencia entre lo exigido por el sistema y lo realizado, entre lo proclamado y la práctica cotidiana, entre objetivos y logros. Todo ello conduce a un aumento de la burocracia y a una falta de adecuación en la formación del docente.

¿Es la sociedad la culpable de que no se lleven a cabo las funciones básicas de la escuela? ¿El profesorado tiene que decantarse por una u otra de las funciones entendidas como transmitir conocimientos y formar ciudadanos, o bien buscar el equilibrio para que se desarrollen ambas y no una en perjuicio de la otra? La respuesta la hallaríamos si el profesorado se hubiera formado adecuadamente, o si la formación, no sólo la reglada, se mantuviera en el ejercicio diario de la docencia. Mientras tanto las responsabilidades quedan fuera, de modo que estas son de carácter institucional (escuelas y facultades de educación) o de carácter individual (profesorado que no las asume).

Para el profesorado hay formación, pero no es una formación diseñada y organizada en torno a los cambios sociales ni a las demandas del sistema educativo; es una formación cuyo objetivo es obtener los puntos necesarios para los concursos de traslados y cuya oferta está dirigida desde las consejerías de educación, si bien poco a poco empiezan a mostrarse otro sistemas de aprendizaje no dirigidos o autodirigidos y que forman parte de estrategias de renovación pedagógica de ciertos colectivos docentes.

Hay profesores que señalan que han aprendido y aprenden mucho viendo trabajar a otros compañeros, hablándoles, imitándoles o colaborando en la organización del 
aula. Son las Ilamadas parejas pedagógicas. Sin embargo, otros profesores entienden que estas prácticas son más usuales y eficaces, sobre todo en centros privados concertados, porque su profesorado se encuentra más implicado, así como por el ideario que sostiene el proyecto educativo de centro que les exige una preparación superior a la exigida en los centros públicos.

Hasta aquí hemos realizado una breve enumeración de responsabilidades (socialización, orientación y motivación del alumnado, utilización de las TIC, relaciones con las familias y formación permanente), que el profesorado asume unas veces con convicción y otras con resignación; pero cuando retomamos su consideración acerca de cuál es la principal función como docentes, impera la idea de que el profesorado es y debe ser un referente para sus alumnos (como recuerdan que fueron algunos maestros en su particular biografía de adolescentes), y de ahí abundan en la necesidad de ser modélicos, es decir, ser un ejemplo en su actividad docente.

Referentes para sus alumnos pese a la heterogeneidad de la gente que compone los claustros, y que son un reflejo o espejo de la heterogeneidad social donde se relacionan los ciudadanos, los cuáles a su vez están presentes en hogares con una composición diversa.

Diversidad y heterogeneidad, diferencia y desigualdad son los conceptos más usuales que el profesorado asume, en este breve recorrido por las funciones y responsabilidades adoptadas como un conjunto de oposiciones que sin embargo deben llevar a cabo en su trabajo como profesionales. Un trabajo que toman para sí pese a su complejidad, de acuerdo a las nuevas expectativas sociales sobre la educación que se han ido depositando en la escuela.

\section{Conclusiones}

Hemos observado que la principal función de la escuela considerada por el profesorado es la guarda y custodia del alumnado matriculado en cada centro; para lo cual determinan que ellos deben ejercer un tipo de autoridad que permita la creación de un clima favorable a la convivencia en el aula sobre el que se pueda llevar a cabo el proceso de enseñanza aprendizaje.

Es una autoridad difusa proveniente (derivada o por extensión), de la sostenida por los padres y las familias, que les permite no sólo la transmisión de los conocimientos marcados en el currículo escolar, sino la asunción de normas básicas de comportamiento y convivencia en el aula, así como las funciones de motivar y orientar a sus alumnos en cuanto tiene que ver con el devenir socio profesional, aspectos que implican aceptar responsabilidades socializadoras.

No es una tarea fácil pues deben competir con otros agentes socializadores como son las familias e Internet, que han irrumpido en el proceso de enseñanza aprendizaje del profesorado, unas a través de la adquisición de niveles socioeducativos iguales o superiores a los del profesorado, y el otro desmotivando al alumnado que encuentra más utilidad funcional en las TIC que en la escuela, con su tradición de pruebas de evaluación mediante exámenes.

La diversidad de contextos y entornos educativos, las diferencias entre etapas educativas o según titularidad de centros, así como la propia heterogeneidad del 
profesorado no permite que éstos se reconozcan como un colectivo profesional pese a disponer de un discurso corporativo que señala pérdidas de derechos y prácticas docentes añadidas (principalmente las burocráticas a través de programaciones, estándares, etc.). Tampoco se sienten un colectivo profesional cuando se relacionan con la administración (salvo en ocasiones puntuales de conflicto), con los equipos directivos y con las familias en su diversidad.

Los roles del profesorado consisten en dirigir, coordinar y orientar a los alumnos para que desarrollen sus propias potencialidades; maduren y adquieran las competencias y las responsabilidades que no les demandan o que no les ofrecen en sus casas o en sus familias, de modo que la responsabilidad del profesorado va más allá de la implementación del currículo escolar asumiendo responsabilidades que se suponen propias de las familias.

La dirección o la orientación que nos indica el profesorado en su discurso, tiene mucho que ver con dirigir, marcar y, en definitiva, transmitir las materias curriculares que tienen que estudiar, pese a que con el desarrollo de las TIC son los propios alumnos los que se erigen en protagonistas de su aprendizaje, no sólo porque el entorno digital de los hogares les ha permitido socializarse en el mismo antes que la propia escuela desarrolle su propia de utilización de las TIC, sino porque el profesorado es ahora quien tiene que potenciar su formación en nuevas relaciones, comunicaciones, proyectos e innovaciones educativas en dicho entorno.

La aceptación de más responsabilidades que las tradicionales de ser un mero transmisor de contenidos, ha permitido cambios en las funciones del profesorado, hasta el punto de señalar como la principal el ser un referente, un ejemplo y un modelo para sus alumnos, con independencia de los resultados académicos, dirigiéndose con más realismo hacia un horizonte de socialización de nuevos ciudadanos.

\section{REFERENCIAS BIBLIOGRÁFICAS}

Andrés, S. y Giró, J. (2016). El papel y la representación del profesorado en la participación de las familias en la escuela. Revista Electrónica Interuniversitaria de Formación del Profesorado, 19(1), 61-71. Recuperado de https://dialnet.unirioja. es/descarga/articulo/5315534.pdf.

Bernard, O. y Llevot, N. (2016). Las relaciones familia-escuela en la formación inicial del profesorado. Opción. Revista de Ciencias Humanas y Sociales, No Extra 7, 959-976.

Bernard, O. y Llevot, N. (2016). El papel de las AMPA en los centros escolares: actuaciones y retos. RASE. Revista de la Asociación de Sociología de la Educación, 9(3), 359-371. Recuperado de https://ojs.uv.es/index.php/RASE/article/ view/8984/8431.

Bolívar, A. (2006). Familia y escuela: dos mundos Ilamados a trabajar en común. Revista de Educación, 339, 119-146.

Consejo Escolar del Estado (2014). La participación de las familias en la educación escolar. Madrid: Ministerio de Educación, Cultura y Deporte. Recuperado de http://www.mecd.gob.es/dctm/cee/publicaciones/estudioparticipacion/estudioparticipacion.pdf?documentld=0901e72b81b45e35. 
Doncel, D. (2012). Magister doloroso. La identidad colectiva del profesorado no universitario desde la perspectiva de las organizaciones sindicales. RASE. Revista de la Asociación de Sociología de la Educación, 5(3), 489-502. Recuperado de https://ojs.uv.es/index.php/RASE/article/view/8340/7933.

Epstein, J. L. (2001). School, Family and Community Partnerships. Preparing Educators and Improving Schools. Boulder: Westview.

Feito, R. (2007). Balance de la participación de los padres en los Consejos Escolares de centro. Participación Educativa, 4, 4-15.

Fernández Enguita, M. (2006). La escuela y la comunidad. En A. García Albaladejo (Coord.), Participación de las familias en la vida escolar: acciones y estrategias (pp. 87-100). Madrid: Ministerio de Educación y Ciencia, Secretaria General de Educación.

Fernández Enguita, M. (2007). Educar es cosa de todos: escuela, familia y comunidad. En J. Garreta (Ed.), La relación familia-escuela (pp. 13-32). Lleida: Ediciones de la Universidad de Lleida.

Fernández Enguita, M. (2016). La educación en la encrucijada. Madrid: Santillana.

Garreta, J. (2003). El espejismo intercultural. La escuela de Cataluña ante la diversidad cultural. Madrid: CIDE.

Garreta, J. (2007). La relación familia-escuela: ¿una cuestión pendiente? En J. Garreta (Ed.), La relación familia-escuela (pp. 9-12). Lleida: Ediciones Universitat de Lleida.

Garreta, J. (2008). La participación de las familias en la escuela pública. Las Asociaciones de Madres y Padres del Alumnado. Madrid: Centro de Investigación y Documentación Educativa (CIDE) y Confederación Española de Asociaciones de Padres y Madres de Alumnos (CEAPA).

Garreta, J. (2009). Escuela y familias inmigradas: relaciones complejas. Revista Complutense de Educación, 20(2), 275-291.

Garreta, J. (Coord.) (2017). Familias y escuelas. Discursos y prácticas sobre la participación en la escuela. Madrid: Pirámide.

Garreta, J. y Llevot, N. (2007). La relación familia - escuela, ¿una cuestión pendiente? En J. Garreta (Ed.), La relación familia-escuela (pp. 9-12). Lleida: Ediciones Universidad de Lleida.

Garreta, J. y Llevot, N. (2015). Family-school communication in Spain: channels and their use. EHQUIDAD, 3, 29-47. http://doi.org/10.15257/ehquidad.2015.0002.

Giró, J. y Andrés, S. (2014). Las familias se suben a la Marea Verde. El papel de madres y padres en las movilizaciones educativas, RASE. Revista de la Asociación de Sociología de la Educación, 7(3), 627-643. Recuperado de https://ojs.uv.es/index. php/RASE/article/view/8746/8289.

Giró, J., Mata, A., Vallespir, J. y Vigo, B. (2014). Familias y escuelas: los diferentes discursos sobre la participación. Ehquidad. International Welfare Policies and Social Work Journal, 2, 65-90. http://doi.org/10.15257/ehquidad.2014.0009.

Gomila, M. A. y Pascual, B. (2015): La participación de las familias en el sistema educativo: la percepción del profesorado en formación, Revista Electrónica Interuniversitaria de Formación del Profesorado, 18(3), 99-112. http://doi.org/10.6018/ reifop.18.3.199321. 
Llevot, N. y Bernard, O. (2015). La participación de las familias en la escuela: factores clave. RASE. Revista de la Asociación de Sociología de la Educación, 8(1), 57-60. Recuperado de https://ojs.uv.es/index.php/RASE/article/view/8761/8304.

Molet, C., Bernard, O. y Mateo, D. (2016). Representations of childhood and education in initial training of teachers identities. En F. Sabaté (Dir.), Conditioned Identities Whised-for and Unwhised-for Identities (pp. 337-356). Bern: Peter Lang.

Pereda, V. (2006). La participación de las familias en los centros educativos. Algunos pasos dados, mucho camino por recorrer. En M. T. López (Ed.), La familia en el proceso educativo (pp. 13-50). Madrid: Cinca.

Pérez-Díaz, V. y Rodríguez, J. C. (2012). Educación y prestigio docente en España: la visión de la sociedad. En V. Pérez-Díaz y J. C. Rodríguez (Dirs.), El prestigio de la profesión docente en España. Percepción y realidad (pp. 33-108). Madrid: Fundación Europea Sociedad y Educación y Fundación Botín.

Rivas, J.I., Leite, A. y Cortés, P. (2011). Paradojas y conflictos entre las culturas del profesorado, las familias y los estudiantes en el contexto escolar. Revista de Educación, 356, 161-183. Recuperado de http://www.revistaeducacion.educacion. es/re356/re356_07.pdf.

San Fabián, J. L. (2001). La participación en la comunidad educativa. En M. Á. Santos Guerra (Coord.), Organización y gestión escolar (pp. 53-70). Barcelona: CISSPraxis.

Usategui, E. y Del Valle, A. I. (2009). Luces y sombras de la función docente desde la mirada del profesorado. Revista Electrónica Interuniversitaria de Formación del Profesorado, 12(2), 19-37. Recuperado de https://dialnet.unirioja.es/descarga/ articulo/3039089.pdf.

Vecina, C., San Román, S. y Doncel, D. (2017). Discurso del profesorado en torno a la sociedad, la familia, la docencia y su función social. Aposta. Revista de Ciencias Sociales, 72, 28-56.

Zapico-Barbeitio, M-H., Martínez, E. y Montero, M. L. (2017). Demandas sociales y formación inicial del profesorado: ¿un callejón sin salida? Profesorado. Revista de currículum y formación del profesorado, 21(1), 80-102. 